\title{
Sensitivity of the CSR Self-Interaction to the Local Longitudinal Charge Concentration of an Electron Bunch
}

\author{
R. Li \\ Jefferson Lab, 12000 Jefferson Ave., Newport News, VA 23606, USA
}

\begin{abstract}
Recent measurements of the coherent synchrotron radiation (CSR) effect carried out at CERN and at Jefferson Lab indicate that the observed emittance growth and energy modulation due to the orbit-curvature-induced bunch self-interaction are sometimes bigger than the results predicted from previous analyses and simulations based on a Gaussian longitudinal charge distribution. In this paper, by performing a model study, we show both analytically and numerically that when the longitudinal bunch charge distribution involves concentration of charges in a small fraction of the bunch length, enhancement of the CSR self-interaction beyond the Gaussian prediction may occur. The level of this enhancement is sensitive to the level of the local charge concentration.
\end{abstract}

\section{INTRODUCTION}

In designs of next-generation accelerators, high-energy electron beams of short bunches with high charges are often transported through magnetic bending systems. For example, in order to obtain the high-peak-current beams required by future linear colliders and short-wavelength free-electron laser (FEL) drivers, bunch compression chicanes consisting of several magnetic bends are often used: after an energy correlation along the bunch length is imposed on the bunch by upstream radiofrequency $(\mathrm{RF})$ cavities, high-charge bunches are transported through a chicane where the final bunch length can be manipulated by employing the energy dependence of path length through the chicane. The Jefferson Lab infrared FEL driver energy recovery recirculator [1] and combiner rings in the compact linear collider (CLIC) design [2] are other examples of high-charge microbunches traversing through magnetic bending systems.

As an electron bunch goes through a bend, each electron emits synchrotron radiation. Coherent synchrotron radiation (CSR) occurs when the radiation wavelength is longer than the bunch length, as a result of the orbitcurvature-induced electromagnetic self-interactions within the bunch. These self-interactions, which are dominated by the collective synchrotron radiation forces at high energy, will induce energy modulation in the bunch, and may cause degradation of beam quality via the energy dependence of particle orbits in the bend. Understanding this CSR effect is crucial for designs of bending systems in future accelerators so as to meet the stringent design requirements on the preservation of small transverse and longitudinal emittances.

Earlier analyses of the CSR self-interaction [3-5] were all based on a rigid-line-charge model. Even though these approaches can be applied for general longitudinal charge distributions, since the analytical results for a Gaussian beam are explicitly given, one usually applies the Gaussian results to estimate the CSR effects using measured or simulated rms bunch lengths. Likewise, a self-consistent simulation [7] was recently developed to study the CSR effect on bunch dynamics for general bunch distributions. However, due to the lack of knowledge of the detailed longitudinal phase-space distributions when simulating an CSR experiment, this simulation is usually carried out assuming a Gaussian phase-space distribution with the rms bunch length derived from bunch length measurements. Recent CSR experiments [8-10] indicate that the measured energy modulation and emittance growth are sometimes bigger than results predicted by these previous analyses and simulations [6,7] using a Gaussian beam. To understand this observed enhancement of the CSR effect, it is instructive to study the CSR self-interaction for a compressed beam which may be highly non-Gaussian. In general, the final distribution of a fully compressed beam is determined by many factors, including the details of the electron source, upstream space-charge interaction, RF structure, wake function and optics - all of which vary with different designs. In this paper, instead of treating the general distribution, we carry out a model study by analyzing and numerically simulating the CSR self-interaction for a bunch compressed by a magnetic chicane with the final bunch length determined only by the RF curvature and beamline optics. This study reveals a general feature of the CSR self-interaction: whenever there is longitudinal charge concentration in a small fraction of a bunch length, enhancement of the CSR effect beyond the Gaussian prediction can occur; moreover, the level of this enhancement is sensitive to the level of the local charge concentration within a bunch. This sensitivity should be given serious consideration in designs of future electron accelerators and when one interprets measurement results of CSR experiments, so as to avoid underestimation of the CSR-induced phase-space dilution for high-charge short electron bunches traversing bending systems. 


\section{BUNCH COMPRESSION OPTICS}

In order to study the CSR self-interaction for a compressed bunch, let us first find the longitudinal charge distribution for our model bunch when it is fully compressed by a chicane (here we assume that the perturbation of bunching process by CSR is negligible because most of the CSR perturbations take place at the end of the chicane when the bunch length reaches its minimum value). We will first study the bunch compression for a beam with zero uncorrelated energy spread $\delta_{\text {un }}=0$, and then generalize the results for $\delta_{\text {un }} \neq 0$. Consider an electron bunch with $N$ electrons. The longitudinal charge density distribution function of the bunch at time $t$ is

$$
\rho(s, t)=\operatorname{Nen}(s, t), \quad \int n(s, t) d s=1,
$$

where $s$ is the longitudinal distance from a reference electron ( $s>0$ for bunch head), and $n(s, t)$ is the longitudinal density distribution of the bunch. As the bunch passes through the chicane, $s$ varies with $t$ for each electron and consequently the density distribution also depends on $t$. Let us identify each electron by the parameter $\mu$, which is the initial longitudinal position $s_{0}$ of the electron at $t=t_{0}$ :

$$
s_{0} \equiv s\left(\mu, t_{0}\right)=\mu \text {. }
$$

At $t=t_{0}$, let the bunch be a line aligned on the design orbit at the entrance of a bunch compression chicane, with a Gaussian longitudinal density distribution and the initial $\mathrm{rms}$ bunch length $\sigma_{s 0}$ :

$$
n\left(s_{0}, t_{0}\right)=n_{0}(\mu)=\frac{1}{\sqrt{2 \pi} \sigma_{s 0}} e^{-\mu^{2} / 2 \sigma_{s 0}^{2}} .
$$

To compress the bunch using the chicane, a linear energy correlation is imposed on the bunch by an upstream RF cavity, along with a slight second-order energy correlation due to the curvature of the RF wave form. With $\delta_{1}$ and $\delta_{2}$ the linear and second-order correlation coefficients $\left(\delta_{1}, \delta_{2}>0\right.$ and $\left.\delta_{2} \ll \delta_{1}\right)$, the relative energy deviation $\delta=\Delta E / E_{0}$ from the design energy $E_{0}$ is then

$$
\delta\left(\mu, t_{0}\right)=-\delta_{1} \frac{\mu}{\sigma_{s 0}}-\delta_{2}\left(\frac{\mu}{\sigma_{s 0}}\right)^{2}
$$

where we assume no uncorrelated energy spread. When the beam propagates to the end of the chicane at $t=t_{f}$, the final longitudinal coordinates of the electrons are

$$
\begin{gathered}
s\left(\mu, t_{f}\right)=s\left(\mu, t_{0}\right)+R_{56} \delta\left(\mu, t_{0}\right)+T_{566}\left[\delta\left(\mu, t_{0}\right)\right]^{2} \\
=s\left(\mu, t_{0}\right)\left(1-\frac{R_{56} \delta_{1}}{\sigma_{s 0}}\right)-\alpha\left[s\left(\mu, t_{0}\right)\right]^{2}
\end{gathered}
$$

where $R_{56}=d L / d \delta$ and $T_{566}=d^{2} L / 2 d \delta^{2}$, with $L$ denoting the path length of an electron through the chicane, and $\alpha \equiv\left(R_{56} \delta_{2}-T_{566} \delta_{1}^{2}\right) / \sigma_{s 0}^{2}$. To obtain the maximum compression of the bunch, one designs the chicane by choosing $R_{56}$, the initial bunch length $\sigma_{s 0}$ and the initial energy correlation $\delta_{1}$ to satisfy

$$
1-R_{56} \delta_{1} / \sigma_{s 0}=0, \quad s_{f} \equiv s\left(\mu, t_{f}\right)=-\alpha\left[s\left(\mu, t_{0}\right)\right]^{2} .
$$

For typical bunch-compression chicanes, one has $R_{56}>0$ and $T_{566}<0$; therefore $\alpha>0$, which implies $s_{f} \leq 0$ from Eq. (7). Using Eqs. (7) and (2), we have

$$
\mu^{2}=-s_{f} / \alpha \quad\left(\alpha>0, s_{f} \leq 0\right)
$$

The final longitudinal density distribution $n\left(s_{f}, t_{f}\right)$ can then be obtained from charge conservation $n_{0}(\mu) d \mu=$ $n\left(s_{f}, t_{f}\right) d s_{f}$ and from Eq. (8), which gives

$$
n\left(s_{f}, t_{f}\right)=\frac{1}{\sqrt{2 \pi} \sigma_{s f}} \frac{e^{s_{f} / \sqrt{2} \sigma_{s f}}}{\sqrt{-s_{f} / \sqrt{2} \sigma_{s f}}} H\left(-s_{f}\right),
$$

where $H(s)$ is the Heaviside step function, and $\sigma_{s f}$ denotes the rms of the final longitudinal distribution 


$$
\sigma_{s f} \equiv \sqrt{\left\langle s_{f}^{2}\right\rangle-\left\langle s_{f}\right\rangle^{2}}=\sqrt{2} \alpha \sigma_{s 0}^{2}
$$

The first moment of the distribution in Eq. (9) is $\left\langle s_{f}\right\rangle=-\sigma_{s f} / \sqrt{2}$.

The distribution in Eq. (9) obtained for $\delta_{\mathrm{un}}=0$ gives unrealistic divergence at $s_{f}=0$. For a realistic beam, uncorrelated energy spread $\delta_{\text {un }}$ should be added to Eq. (4) (here we assume $\delta_{\text {un }}$ has a Gaussian distribution with $\left\langle\delta_{\text {un }}\right\rangle=0$, and rms width $\delta_{\text {un }}^{\mathrm{rms}}$ ). As a result, the final longitudinal phase space distribution can be obtained by combining Eqs. (4), (7) and (10), yielding

$$
s_{f} \simeq-\left(\sigma_{s f} / \sqrt{2} \delta_{1}^{2}\right) \delta^{2}+R_{56} \delta_{\text {un }}
$$

and the final effective rms bunch length becomes

$$
\sigma_{s f}^{\mathrm{eff}} \equiv \sqrt{\left\langle s_{f}^{2}\right\rangle-\left\langle s_{f}\right\rangle^{2}}=\sqrt{\sigma_{s f}^{2}+R_{56}^{2}\left\langle\delta_{\mathrm{un}}^{2}\right\rangle}
$$

with $\sigma_{s f}$ given by Eq. (10). For example, when $\sigma_{s 0}=1.26 \mathrm{~mm}, R_{56}=45 \mathrm{~mm}, \delta_{1}=0.028$, the compression condition Eq. (7) is satisfied. With $\alpha=0.08 \mathrm{~mm}^{-1}$, Eq. (10) gives the final compressed bunch length $\sigma_{s f}=0.18 \mathrm{~mm}$. An example of the longitudinal phase-space distribution described by Eq. (11) is shown in Fig. 1.

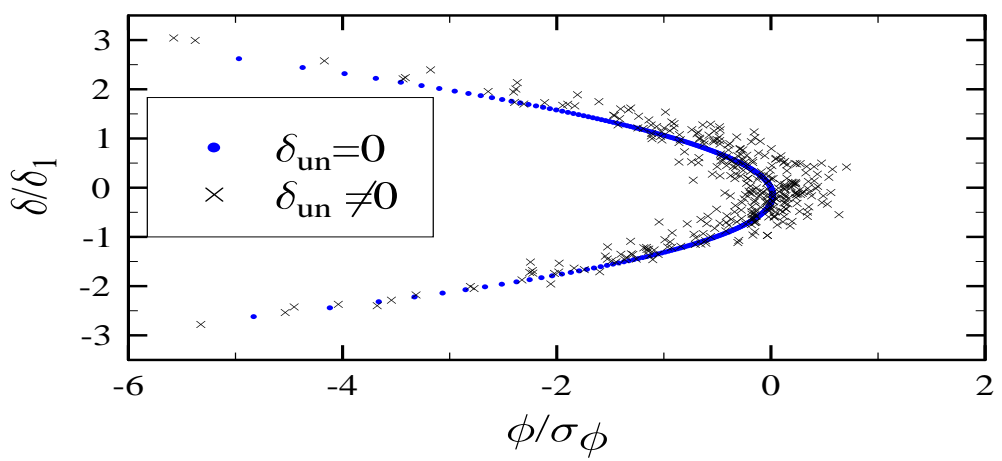

FIG. 1. Example of the longitudinal phase-space distribution for a compressed beam with RF curvature effect.

Next, we consider a rigid-line bunch on a circular orbit of radius $R$, with the longitudinal density distribution function obtained from Eq. (9) for a compressed bunch

$$
\lambda_{0}^{\mathrm{cmpr}}(\phi)=\frac{1}{\sqrt{2 \pi} \sigma_{\phi}} \frac{e^{\phi / \sqrt{2} \sigma_{\phi}}}{\sqrt{-\phi / \sqrt{2} \sigma_{\phi}}} H(-\phi),
$$

where $\phi=s / R$ and $\sigma_{\phi}=\sigma_{s} / R$, with $s$ denoting the longitudinal distance from reference electron and $\sigma_{s}$ the rms bunch length. To account for the effect of the uncorrelated energy spread in Eq. (11), which gives the compressed bunch an intrinsic width, we let the compressed bunch be a continuous distribution of macroparticles with their centroid distribution described by Eq. (13), while each macroparticle is a line Gaussian distribution with the density function

$$
\lambda_{m}(\phi)=e^{-\phi^{2} / 2 \sigma_{m \phi}^{2}} / \sqrt{2 \pi} \sigma_{m \phi}, \quad \sigma_{m \phi}=\frac{R_{56} \delta_{\mathrm{un}}^{\mathrm{rms}}}{R},
$$

where $\sigma_{m \phi}$ is the angular rms size for each macroparticle. Hence the overall distribution for a compressed bunch is

$$
\lambda^{\mathrm{cmpr}}(\phi)=\int_{-\infty}^{\infty} \lambda_{0}^{\mathrm{cmpr}}(\phi-\varphi) \lambda_{m}(\varphi) d \varphi
$$

Denoting $a$ as the ratio of the intrisic width to the rms bunch length (here we are interested in $0<a<1$ )

$$
a=\frac{\sigma_{m \phi}}{\sigma_{\phi}}=\frac{R_{56} \delta_{\mathrm{un}}^{\mathrm{rms}}}{R \sigma_{\phi}}
$$


we find $\lambda^{\mathrm{cmpr}}(\phi)$ in Eq. (15) becomes

$$
\lambda^{\mathrm{cmpr}}(\phi)=\frac{2^{1 / 4}}{4 \sigma_{\phi} \sqrt{a}} \operatorname{Exp}\left(-\frac{\phi^{2}}{2 a^{2} \sigma_{\phi}^{2}}+\frac{u^{2}}{4}\right) \sqrt{|u|}\left\{I_{-\frac{1}{4}}\left(\frac{u^{2}}{4}\right)+[H(-u)-H(u)] I_{\frac{1}{4}}\left(\frac{u^{2}}{4}\right)\right\}
$$

where $u=\frac{a}{\sqrt{2}}+\frac{\phi}{a \sigma_{\phi}}$ and $I_{\nu}(x)$ is the modified Bessel function. The asymptotic forms of $\lambda^{\mathrm{cmpr}}(\phi)$ in Eq. (17) can be obtained from the asymptotic behavior of $I_{\nu}(x)$ :

$$
\lambda^{\mathrm{cmpr}}(\phi)=\frac{1}{2^{1 / 4} \sigma_{\phi} \sqrt{\pi a|u|}} \begin{cases}\frac{1}{\sqrt{2}} e^{-\phi^{2} / 2 a^{2} \sigma_{\phi}^{2}} & (u>0,|u| \gg 1), \\ e^{a^{2} / 4} e^{-|\phi| / \sqrt{2} \sigma_{\phi}} & (u<0,|u| \gg 1) .\end{cases}
$$

Note $a=0$ gives $\lambda^{\mathrm{cmpr}}(\phi)=\lambda_{0}^{\mathrm{cmpr}}(\phi)$. From Eq. (15), one finds the rms angular width for $\lambda^{\mathrm{cmpr}}(\phi)$

$$
\sigma_{\phi}^{\mathrm{eff}} \equiv \sqrt{\left\langle\phi^{2}\right\rangle-\langle\phi\rangle^{2}}=\sigma_{\phi} \sqrt{1+a^{2}}
$$

which agrees with Eq. (12). Fig. 2 shows good agreement of the analytical result of the longitudinal density distribution in Eq. (17) with the numerical result for the example given in Fig. 1.

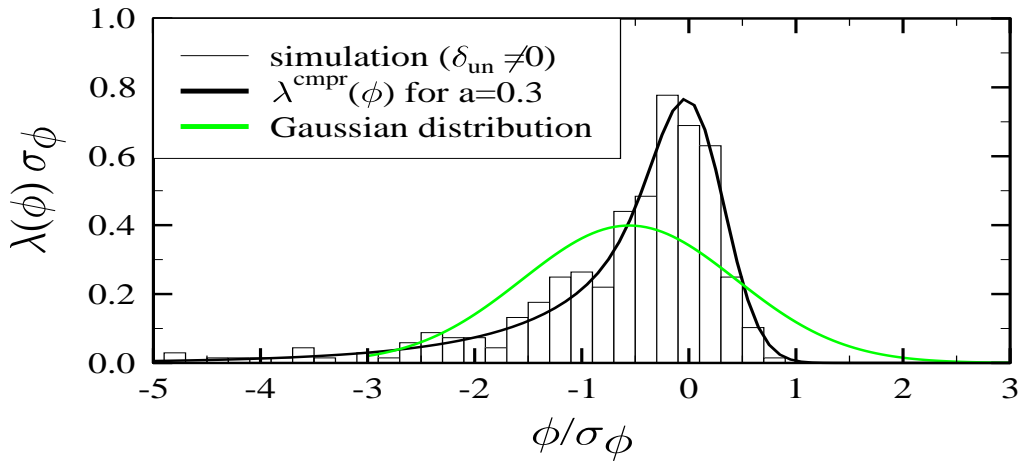

FIG. 2. Analytical and numerical result of the longitudinal density distribution for the example shown in Fig. 1, with $a$ defined in Eq. (16).

\section{CSR SELF-INTERACTION FOR A COMPRESSED BUNCH}

To study the CSR self-interaction for the rigid-line bunch in Eq. (17) under a steady-state circular motion, let us first enlist the formulae established by earlier analyses [3-5]. The longitudinal collective force via space charge and CSR interaction is derived from the scalar and vector potentials $\Phi$ and $\mathbf{A}$,

$$
F_{\theta}(\phi)=\frac{e}{\beta c} \frac{\partial}{\partial t}(\Phi-\boldsymbol{\beta} \cdot \mathbf{A})=\frac{-N e^{2}}{R^{2}} \int_{0}^{\infty} \frac{1-\beta^{2} \cos \theta}{2 \sin (\theta / 2)} \frac{\partial}{\partial \phi} \lambda(\phi-\varphi) d \varphi
$$

where $\boldsymbol{\beta}=\mathbf{v} / c, \beta=|\boldsymbol{\beta}|, \gamma=1 / \sqrt{1-\beta^{2}}$, and $\theta$ is an implicit function of $\varphi$ via the retardation relation $\varphi=$ $\theta-2 \beta \sin (\theta / 2)$. In this paper, we treat only the high-energy case when $\gamma \gg \theta^{-1}$ and $\theta \simeq 2(3 \varphi)^{1 / 3}$. In this case $F_{\theta}(\phi)$ is dominated by the radiation interaction:

$$
F_{\theta}(\phi) \simeq \frac{-N e^{2}}{R} \int_{0}^{\infty} \frac{\beta^{2} \sin \frac{\theta}{2}}{1-\beta \cos \frac{\theta}{2}} \frac{\partial}{\partial \phi} \lambda(\phi-\varphi) d \varphi \simeq \frac{-2 N e^{2}}{3^{1 / 3} R^{2}} \int_{0}^{\infty} \varphi^{-1 / 3} \frac{\partial}{\partial \phi} \lambda(\phi-\varphi) d \varphi .
$$

The radiation power due to the radiation interaction is

$$
P=-N \int F_{\theta}(\phi) \lambda(\phi) d \phi
$$


Results for the longitudinal collective force and the radiation power for a rigid-line Gaussian beam are [3-5]:

$$
\begin{aligned}
& \lambda^{\text {Gauss }}(\phi)=\frac{1}{\sqrt{2 \pi} \sigma_{\phi}} e^{-\phi^{2} / 2 \sigma_{\phi}^{2}} \quad\left(\sigma_{\phi} \gg \frac{1}{\gamma^{3}}\right), \\
& F_{\theta}^{\text {Gauss }}(\phi) \simeq F_{g} \int_{0}^{\infty} \frac{\left(\phi / \sigma_{\phi}-\phi_{1}\right)}{\phi_{1}^{1 / 3}} e^{-\left(\phi / \sigma_{\phi}-\phi_{1}\right)^{2} / 2} d \phi_{1} \quad \text { with } \quad F_{g}=\frac{2 N e^{2}}{3^{1 / 3} \sqrt{2 \pi} R^{2} \sigma_{\phi}^{4 / 3}}, \\
& P^{\text {Gauss }} \simeq \frac{N^{2} e^{2}}{R^{2} \sigma_{\phi}^{4 / 3}} \frac{3^{1 / 6} \Gamma^{2}(2 / 3)}{2 \pi} .
\end{aligned}
$$

where $\Gamma(x)$ is the Gamma function.

Next, we analyze the CSR self-interaction for a rigid-line bunch with the density function given in Eq. (17) under the condition $\sigma_{\phi}>\sigma_{m \phi} \gg \gamma^{-3}$. We proceed by first find CSR force for a compressed bunch with $a=0$ and then extend this result to cases with $a>0$. Applying Eq. (13) to Eq.(2), we obtain the longitudinal collective force on a compressed bunch with zero width as a result of radiation interaction:

$$
F_{\theta 0}^{\mathrm{cmpr}}(\phi) \simeq \frac{-2 N e^{2}}{3^{1 / 3} R^{2}} \int_{0}^{\infty} \varphi^{-1 / 3} \frac{\partial}{\partial \phi} \lambda_{0}^{\mathrm{cmpr}}(\phi-\varphi)=-2^{1 / 4} F_{g} d G(y) / d y \quad\left(y=\phi / \sigma_{\phi}\right),
$$

with $F_{g}$ given in Eq. (5), and

$$
G(y)=H(-y) e^{-|y| / \sqrt{2}}|y|^{1 / 6} \Gamma\left(\frac{2}{3}\right) \Psi\left(\frac{2}{3}, \frac{7}{6} ; \frac{|y|}{\sqrt{2}}\right)+H(y) y^{1 / 6} \Gamma\left(\frac{1}{2}\right) \Psi\left(\frac{1}{2}, \frac{7}{6} ; \frac{y}{\sqrt{2}}\right),
$$

where $\Psi(a, \gamma, z)$ is the degenerate hypergeometric function

$$
\Psi(\alpha, \gamma ; z)=\frac{1}{\Gamma(\alpha)} \int_{0}^{\infty} e^{-z t} t^{\alpha-1}(1+t)^{\gamma-\alpha-1} d t .
$$

The steady-state CSR longitudinal force for a compressed bunch with nonzero width $a \neq 0$ ( $a$ given in Eq. (16)) can then be obtained using Eqs. (2), (15) and (7), yielding

$$
F_{\theta}^{\mathrm{cmpr}}(\phi)=\int_{-\infty}^{\infty} F_{\theta 0}^{\mathrm{cmpr}}(\varphi) \lambda_{m}(\phi-\varphi) d \varphi=\frac{2^{1 / 4} F_{g}}{\sqrt{2 \pi} a^{5 / 6}} f\left(\frac{\phi}{a \sigma_{\phi}} ; a\right)
$$

with

$$
f(y ; a)=\int_{-\infty}^{\infty} G(a x)(y-x) e^{-(y-x)^{2} / 2} d x
$$

Similarly, the radiation power can also be obtained for the compressed bunch using Eq. (3) with $\lambda^{\mathrm{cmpr}}(\phi)$ in Eq. (17) and $F_{\theta}^{\mathrm{cmpr}}(\phi)$ in Eq. (10), which gives

$$
\frac{P^{\mathrm{cmpr}}}{P^{\mathrm{Gauss}}} \simeq 0.75 \frac{I(a)}{a^{5 / 6}}, \quad \text { with } \quad I(a)=-\int_{-\infty}^{\infty} f\left(\frac{\phi}{a \sigma_{\phi}} ; a\right) \lambda^{\mathrm{cmpr}}(\phi) d \phi .
$$

Numerical integration shows that $|f(y ; a)|_{\max }$ - the maximum of $|f(y ; a)|$ for fixed $a$ — is insensitive to $a$ for $0<a<1$, as depicted in Fig. 3. As a result, for a compressed bunch with fixed $\sigma_{\phi}$, we found from Eq. (10) the amplitude of the CSR force $F_{\theta}^{\mathrm{cmpr}}(\phi)$ varies with $a^{-5 / 6}$. Therefore in contrast to the well-known scaling law $R^{-2 / 3} \sigma_{s}^{-4 / 3}$ for the amplitude of the longitudinal CSR force for a Gaussian beam, a bunch described by Eq. (17) has

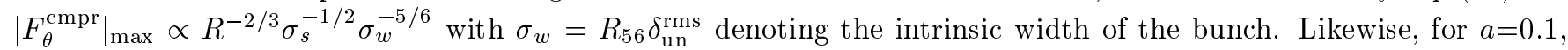
0.2 , and 0.5 , we found from numerical integration that $I(a) \simeq 0.76,0.90$ and 1.02 respectively, and correspondingly $P^{\mathrm{cmpr}} / P^{\text {Gauss }} \simeq 3.9,2.6$ and 1.4. This dependence of the amplitude of the CSR force and power on the intrinsic width of the bunch for a fixed rms bunch length manifests the sensitivity of the enhancement of the CSR effect on the local charge concentration in a longitudinal charge distribution. 


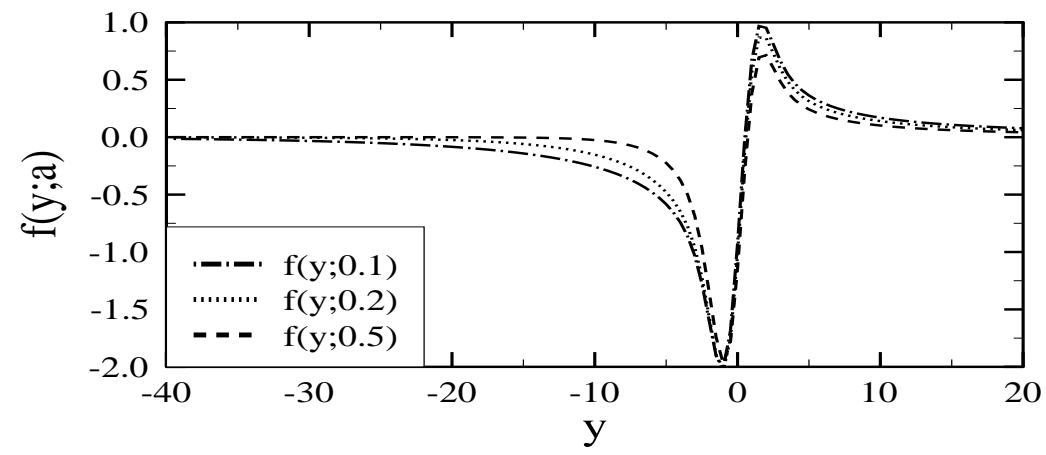

FIG. 3. $f(y ; a)$ vs. $y$ for $a=0.1,0.2$, and 0.5 .

In Figs. 4 and 5, we plot the longitudinal density function for various charge distributions with the same rms bunch lengths (except the $\sqrt{1+a^{2}}$ factor in Eq. (19)), and the longitudinal CSR collective forces associated with the various distributions. The amplitude of $F_{\theta}^{\mathrm{cmpr}}$ in Fig. 4 agrees with the $a^{-5 / 6}$ dependence in Eq. (10). Good agreement of the analytical result in Eq. (10) with the simulation result [7] for the CSR force along the example distribution in Fig. 1 is shown in Fig. 6. Figs. 4 and 5 show clearly that the more locally concentrated are the charges than the smooth behavior of a Gaussian beam, the more enhancement will result from the CSR self-interaction forces. This general feature of the CSR interaction and emission is not limited to the RF curvature effect described in this paper [11]; furthermore, our simulation shows that the enhancement also occurs in the transient regime. Comparing to a Gaussian beam, the local charge concentration in a small fraction of the bunch length can lead to a bigger emittance growth for a compressed or over-compressed beam in a chicane, and a larger energy spread can be observed if the compressed beam is sent to a spectrometer magnet downstream of the chicane, where the beam can further interact with itself via CSR. The frequency domain analysis and the effect of interaction with off-axis particles will be discussed elsewhere.

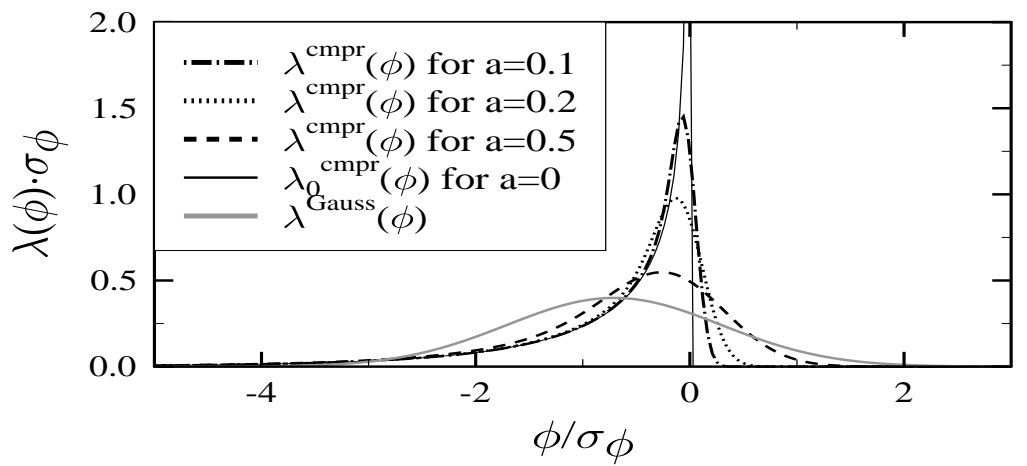

FIG. 4. Longitudinal charge distribution for a compressed beam with relative width described by $a$, compared with a Gaussian distribution. All the distributions here have the same angular rms size $\sigma_{\phi}$.

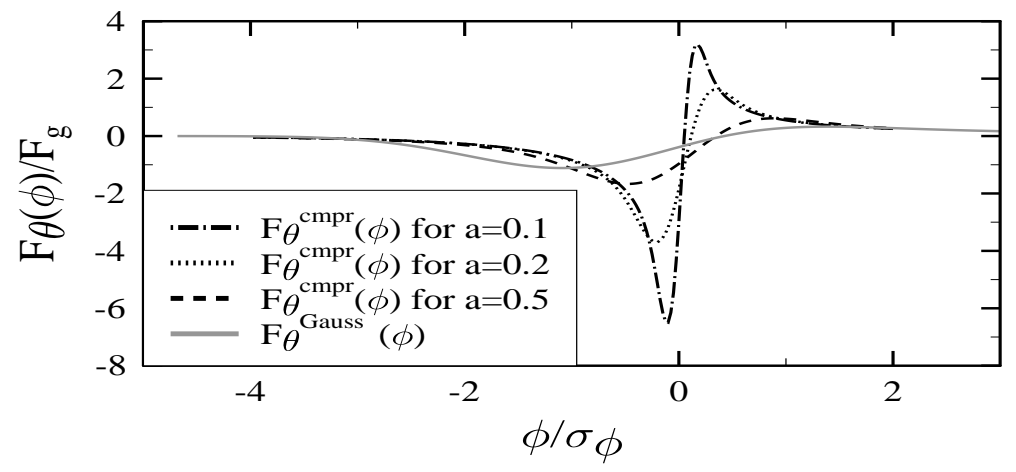


FIG. 5. Longitudinal CSR force along the bunch for various charge distributions illustrated in Fig. 4.

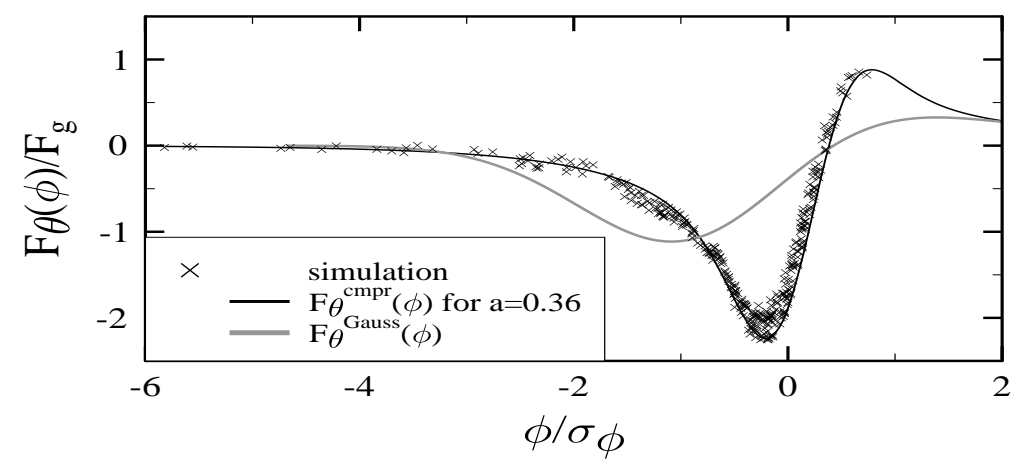

FIG. 6. Comparison of the analytical and numerical results of the longitudinal CSR force along the bunch for the example distributions illustrated in Figs. 1 and 2. Because of the finite macroparticle size used for the force simulation, the width parameter $a$ is slightly enlarged from its value in Fig. 2. The transverse rms size $\sigma_{x}$ used in the simulation is $\sigma_{x} \simeq 3 \sigma_{s}$.

\section{ACKNOWLEDGMENTS}

This work was inspired by the CSR measurement led by H. H. Braun at CERN, and by discussions with T. O. Raubenheimer, R. Corsini, H. Braun, T. Limburg and L. Groening during the measurement. The author is also grateful for valuable discussions with J. J. Bisognano, and with P. Piot, C. Bohn, D. Douglas, G. Krafft and B. Yunn for the CSR measurement at Jefferson Lab. This work was supported by the U.S. Dept. of Energy under Contract No. DE-AC05-84ER40150.

[1] C. L. Bohn, Proc. of the 1997 Part. Accel. Conf.(IEEE No. 97CH36167), Vol. 1, p. 909. D. D. Douglas, Proc. of the 1999 Part. Accl. Conf.(IEEE No. 99CH36366), Vol. 2, p. 1351.

[2] J. P. Delahaye et al., CERN/PS 98-009 (LP), (1998) (unpublished).

[3] Y. S. Derbenev et al., DESY Report No. TESLA-FEL-95-05, 1995 (unpublished).

[4] J. Murphy et al., Part. Accel. 57, 9 (1997).

[5] E. L. Saldin et al., DESY-TESLA-FEL-96-14, (1996) (unpublished).

[6] M. Dohlus and T. Limberg, Proc. of the 18th International Free Electron Laser Conference, Rome, 1996 (unpublished).

[7] R. Li, Nucl. Instrum. Meth. Phys. Res. A 429, 310 (1998).

[8] H. H. Braun et al., Phys. Rev. Lett. 84, 658 (2000).

[9] L. Groening et al., to be presented at the 2000 European Part. Accel. Conf., 2000.

[10] P. Piot, et al., to be presented at the 2000 European Part. Accel. Conf., 2000.

[11] G. A. Krafft, Proc. of the 3rd European Workshop on Beam Diagnostics and Instrumentation for Particle Accelerators, DIPAC97, LNF-97/048,LNF-INFN Frascati, 45 (1997). 